\title{
Development of Aluminum LEKIDs for Balloon-Borne Far-IR Spectroscopy
}

\author{
S. Hailey-Dunsheath ${ }^{1}$. A. C. M. Barlis ${ }^{2}$.

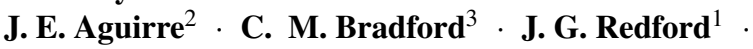 \\ T. S. Billings ${ }^{2}$ - H. G. LeDuc ${ }^{3}$. C. M. McKenney ${ }^{4}$. \\ M. I. Hollister ${ }^{5}$
}

the date of receipt and acceptance should be inserted later

\begin{abstract}
We are developing lumped-element kinetic inductance detectors (LEKIDs) designed to achieve background-limited sensitivity for far-infrared (FIR) spectroscopy on a stratospheric balloon. The Spectroscopic Terahertz Airborne Receiver for Far-InfraRed Exploration (STARFIRE) will study the evolution of dusty galaxies with observations of the [CII] $158 \mu \mathrm{m}$ and other atomic fine-structure transitions at $z=0.5-1.5$, both through direct observations of individual luminous infrared galaxies, and in blind surveys using the technique of line intensity mapping. The spectrometer will require large format $(\sim 1800 \mathrm{de}-$ tectors) arrays of dual-polarization sensitive detectors with NEPs of $1 \times 10^{-17} \mathrm{~W} \mathrm{~Hz}^{-1 / 2}$. The low-volume LEKIDs are fabricated with a single layer of aluminum ( $20 \mathrm{~nm}$ thick) deposited on a crystalline silicon wafer, with resonance frequencies of $100-250 \mathrm{MHz}$. The inductor is a single meander with a linewidth of $0.4 \mu \mathrm{m}$, patterned in a grid to absorb optical power in both polarizations. The meander is coupled to a circular waveguide, fed by a conical feedhorn. Initial testing of a small array prototype has demonstrated good yield, and a median NEP of $4 \times 10^{-18} \mathrm{~W} \mathrm{~Hz}^{-1 / 2}$.
\end{abstract}

Keywords Kinetic Inductance Detector, Aluminum, Far-Infrared Spectroscopy, Balloon

\section{STARFIRE Instrument}

Understanding the formation and evolution of galaxies is one of the foremost goals of astrophysics and cosmology today. The cosmic star formation rate rose dramatically from early times to a peak at approximately half the present age of the universe (at redshift $z \sim 1$ ), with much of the activity occurring in highly dust-obscured systems. A variety of atomic and molecular diagnostic lines are present in the far-infrared (FIR) that are largely unaffected by dust, and can provide insight into the conditions of star formation at the cosmic peak. STARFIRE (the Spectroscopic Terahertz Airborne Receiver for Far-InfraRed Exploration) is

1: California Institute of Technology, Mail Code 301-17, 1200 E. California Blvd., Pasadena, CA 91125, USA; E-mail: haileyds@caltech.edu

2: University of Pennsylvania Department of Physics \& Astronomy, 209 S 33rd St., Philadelphia, PA, 19104, USA

3: Jet Propulsion Laboratory, 4800 Oak Grove Drive, Pasadena, CA, 91109, USA

4: National Institute of Standards and Technology, 325 Broadway, Boulder, CO, 80305

5: Fermi National Accelerator Laboratory, PO Box 500, Batavia IL 60510 
Fig. 1: Electromagnetic simulation of the coupling efficiency of a $\mathrm{TE}_{11}$ mode from the circular waveguide into the aluminum absorber, for sheet impedances of $2 \Omega / \square$ (purple) and $1 \Omega / \square$ (red), and a $27 \mu \mathrm{m}$ backshort. Half power frequencies of the testbed bandpass filter are also shown (dashed). The band-averaged coupling efficiencies are $94 \%$ and $88 \%$ for $2 \Omega / \square$ and $1 \Omega / \square$, respectively, and drop to $22 \%$ and $20 \%$ with no backshort. (Color figure online.)

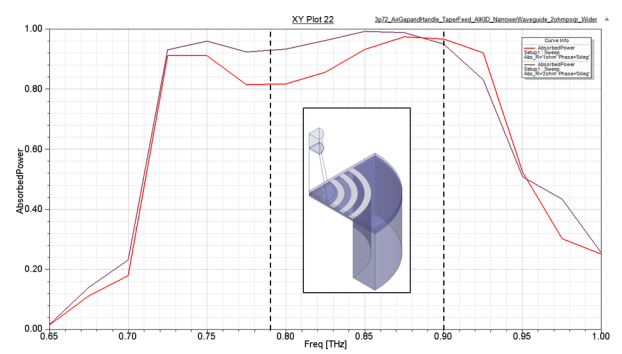

designed to study the interstellar medium (ISM) of galaxies from $0.5<z<1.5$, primarily in the [CII] $158 \mu \mathrm{m}$ line, and also in cross-correlation with the [NII] $122 \mu \mathrm{m}$ transition. STARFIRE will be capable of making a high significance measurement of the [CII] power spectrum in at least 4 redshift bins, and of measuring the $[\mathrm{CII}] \times[\mathrm{NII}]$ power spectrum at $z \sim 1$ [10]. STARFIRE will also be able to detect emission lines in a blind survey, and by correlating with known optical galaxies measure the [CII] luminosity of this population as well.

STARFIRE is able to achieve its substantial increase in performance - better than the airborne instruments on SOFIA or the space-borne Herschel-SPIRE FTS - by using dispersive spectroscopy to lower the photon noise per detector, and by taking advantage of the considerably lower atmospheric background at balloon rather than aircraft altitudes. STARFIRE will field two large format ( $\sim 1800$ detectors each) arrays of dual-polarization sensitive detectors with NEPs below the typical photon NEP of $1.5 \times 10^{-17} \mathrm{~W} \mathrm{~Hz}^{-1 / 2}$. STARFIRE serves as a technology advancement platform for the Origins Space Telescope [9], and detector development is currently funded by NASA [2].

\section{Detector Design and Fabrication}

STARFIRE will deploy arrays of kinetic inductance detectors (KIDs) with the same singlelayer architecture developed for the MAKO camera [8]. A single $20 \mathrm{~nm}$ thick aluminum layer forms both the inductor and interdigitated capacitor, which are designed to achieve $100-250 \mathrm{MHz}$ readout frequencies. To maximize responsivity the absorber is low volume $\left(V=38 \mu \mathrm{m}^{3}\right)$, and couples to incident radiation with a circular waveguide fed with a conical feedhorn. The waveguide design includes a flare at the bottom of the waveguide and a lithographically patterned choke structure to help eliminate conversion into substrate modes. The final pixel will be fabricated on a SOI wafer and will have a $27 \mu \mathrm{m}$ thick backshort, created by etching from the backside to a buried oxide layer, then depositing gold. Electromagnetic simulations indicate band-averaged coupling efficiencies of $\approx 90 \%$ with the backshort in place, and $\approx 20 \%$ without (Figure 1). Initial testing of a device with the backshort fabricated indicate the presence of the gold has no noticeable impact on the resonator $Q$.

The inductor/absorber is a single meander of $0.4 \mu \mathrm{m}$ wide aluminum, patterned to provide an optimal impedance match to the waveguide. The meander effectively couples as a mesh to both polarizations by allowing the various segments of meander line to come close enough to one another at the corners to create capacitive shorts at the optical frequencies. This is achieved with a $0.3 \mu \mathrm{m}$ gap and a $0.6 \mu \mathrm{m}$ overlap length for each of the intersections (Figure 2). 


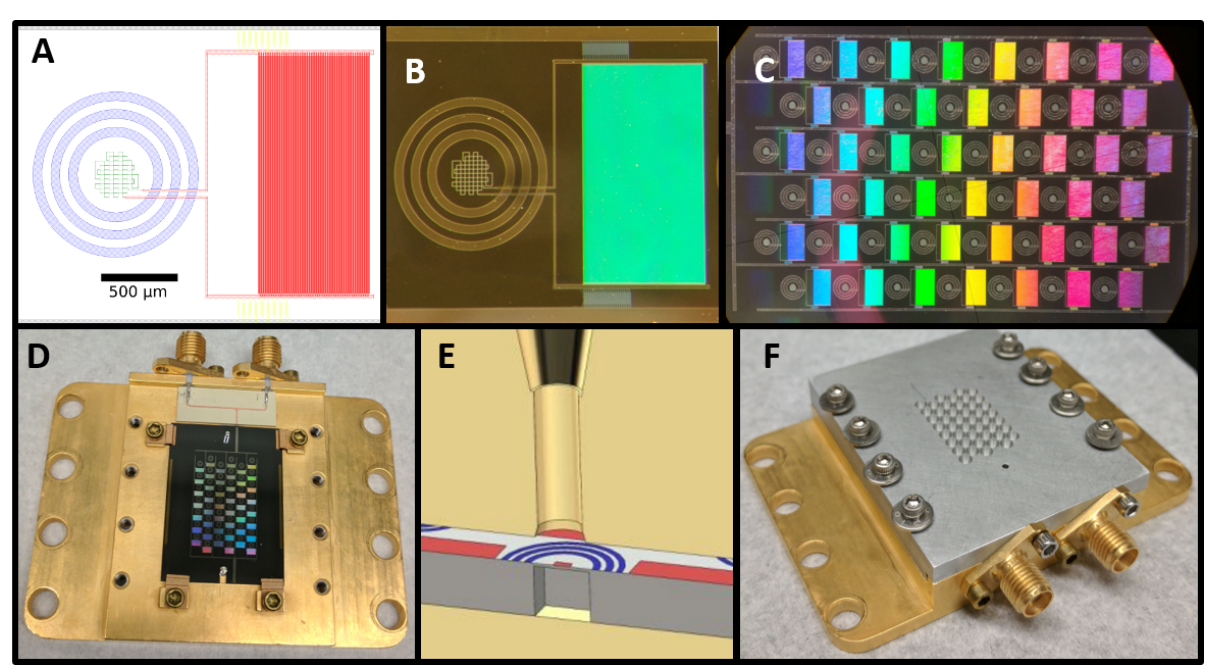

Fig. 2: (A) Diagram of the mask layout for a single resonator. The meandered inductor (green) is surrounded by an optical choke structure (blue). An interdigitated capacitor (red) sets the resonance frequency of the pixel, and two coupling capacitors (yellow) couple microwave signal onto microstrip feedlines. (B) A microscope image of a single pixel as fabricated. All pixel elements of the prototype array are patterned out of $40 \mathrm{~nm} \mathrm{Al} \mathrm{film.} \mathrm{(C)} \mathrm{A} \mathrm{microscope} \mathrm{image} \mathrm{of} \mathrm{the} \mathrm{45-pixel} \mathrm{prototype} \mathrm{array,} \mathrm{as} \mathrm{fabricated.} \mathrm{(D)} \mathrm{The} \mathrm{fabricated}$ array in its enclosure. The back side of the die is bare silicon, and lies flat on the gold-plated package surface. The full size of the die is $30 \mathrm{~mm} \times 22 \mathrm{~mm}$. (E) A CAD model of the detector package. The optical power is coupled into a feedhorn, and travels through a circular waveguide that is terminated by the inductor of the LEKID. The final design will have a backside etch providing a backshort. $(\mathrm{F})$ The prototype feedhorn block installed above the 45-pixel array. (Color figure online.)

\section{Device Testing}

We have cryogenically tested a 45-pixel prototype detector array, fabricated in the JPL Microdevices Laboratory. To measure the performance of detectors with different film thicknesses we fabricated wafers with both $20 \mathrm{~nm}$ and $40 \mathrm{~nm}$ thick aluminum, and conducted the initial testing using an array with a $40 \mathrm{~nm}$ thick film. This results in a larger inductor volume $\left(V=76 \mu \mathrm{m}^{3}\right)$ and higher resonance frequencies than expected for the full STARFIRE array, which will use a $20 \mathrm{~nm}$ thick film. Measurements of the sheet impedances of these films are in preparation. The array is cooled by a ${ }^{3} \mathrm{He}$ sorption fridge to a base temperature of $210 \mathrm{mK}$, and exposed to a cryogenic blackbody for optical testing. We use two metal-mesh filters mounted on the detector package to define the optical band: a bandpass filter transmitting over $\approx 790-900 \mathrm{GHz}$, and a $1000 \mathrm{GHz}$ cutoff low-pass filter. We use a ROACH-based readout system originally developed for use with MAKO [8]. The prototype device had a total yield of $89 \%$ (40/45 resonators), but we focus our analysis on the 11 resonators with resonances below $250 \mathrm{MHz}$.

\subsection{Dark Measurements}

Our first step is to characterize the detectors dark, with the feedhorns blanked off. We measure the resonator frequency, $Q$, and noise as a function of stage temperature. We model our resonators following the standard application of Mattis-Bardeen theory, along with the 

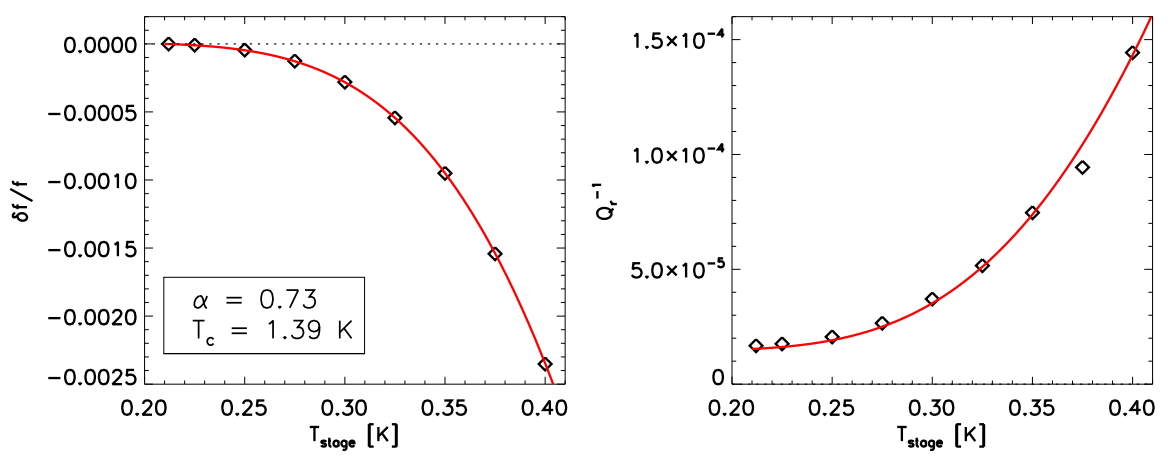

Fig. 3: Fractional frequency shift and $Q_{r}^{-1}$ vs. $T_{\text {stage }}$ for a dark measurement, along with fits for $\alpha$ and $T_{c}$. Models are $x=x_{\mathrm{MB}}+\delta x$ for a zero temperature offset $\delta x$, and $Q_{r}^{-1}=Q_{\mathrm{MB}}^{-1}+Q_{0}^{-1}$, with $Q_{0}^{-1}$ a fixed term that includes the resonator coupling $Q$ and a limiting inductor internal $Q$. (Color figure online.)

assumption that the quasiparticle lifetime depends on the quasiparticle number density as $\tau_{\mathrm{qp}}=\tau_{\max }\left(1+n_{\mathrm{qp}} / n^{*}\right)^{-1}$, for constants $\tau_{\max }$ and $n^{*}[5,11]$. For a general temperature and absorbed optical power $\left(P_{\mathrm{abs}}\right), n_{\mathrm{qp}}$ may be written as:

$$
n_{\mathrm{qp}}=-n^{*}+\left[\left(n^{*}+n_{\mathrm{th}}\right)^{2}+\frac{2 n^{*} \eta_{\mathrm{pb}} P_{\mathrm{abs}} \tau_{\mathrm{max}}}{\Delta_{0} V}\right]^{0.5},
$$

where $n_{\mathrm{th}}=2 N_{0} \sqrt{2 \pi k_{B} T \Delta_{0}} \exp \left(-\Delta_{0} / k_{B} T\right)$ is the quasiparticle density in thermal equilibrium, $\eta_{\mathrm{pb}}$ is the pair-breaking efficiency, $\Delta_{0}=1.76 k_{B} T_{c}$ is the gap energy, $V$ is the inductor volume, and we adopt a density of states of $N_{0}=1.72 \times 10^{10} \mu \mathrm{m}^{-3} \mathrm{eV}^{-1}$ [5]. The fractional frequency shift and the internal $Q$ of the inductor are then written as:

$$
x_{\mathrm{MB}}=-\frac{\alpha \gamma S_{2}}{4 N_{0} \Delta_{0}} n_{\mathrm{qp}} \quad \text { and } \quad Q_{\mathrm{MB}}^{-1}=\frac{\alpha \gamma S_{1}}{2 N_{0} \Delta_{0}} n_{\mathrm{qp}},
$$

where $\gamma=1$ is appropriate for the thin films used here. We use the standard expressions for $S_{1}$ and $S_{2}$ [11], but in place of the physical temperature of our devices we substitute an effective electron temperature, obtained by inverting $n_{\mathrm{th}}(T)$ for $n_{\mathrm{qp}}$ [3]. This modified approach becomes important when the absorbed power is nonzero, and $n_{\mathrm{qp}}>n_{\mathrm{th}}$. In Figure 3 we show measurements of $x$ and $Q_{r}^{-1}$ as a function of $T_{\text {stage }}$ for one resonator, along with fits for $\alpha$ and $T_{c}$. The resonators are well-characterized by Equations 1 and 2 with median values of $\alpha=0.74$ and $T_{c}=1.39 \mathrm{~K}$.

We measure the fractional frequency noise $\left(S_{\mathrm{xx}}\right)$ at each stage temperature, driving the resonators $\sim 3 \mathrm{~dB}$ below bifurcation to minimize amplifier noise. In all measurements presented here the amplifier noise is measured in the dissipation direction, and subtracted off from the measured $S_{\mathrm{xx}}$ (Figure 4). Our general model for the white noise in our KIDs combines photon generation noise, thermal generation noise, and recombination (of all quasiparticles) noise, along with a fixed term $\left(S_{\mathrm{xx}, 0}\right)$ representing additional noise sources, assumed to be independent of temperature and optical loading:

$$
S_{x x}=\left(\frac{\alpha \gamma S_{2}}{4 N_{0} \Delta_{0}}\right)^{2}\left[\left(\frac{\eta_{\mathrm{pb}} \tau_{\mathrm{qp}}}{\Delta_{0} V}\right)^{2} 2 h v P_{\mathrm{abs}}\left(1+n_{\gamma}\right)+\frac{4\left(\tau_{\mathrm{qp}}\right)^{2}}{V^{2}}\left(\Gamma_{\mathrm{th}}+\Gamma_{r}\right)\right]+S_{x x, 0},
$$



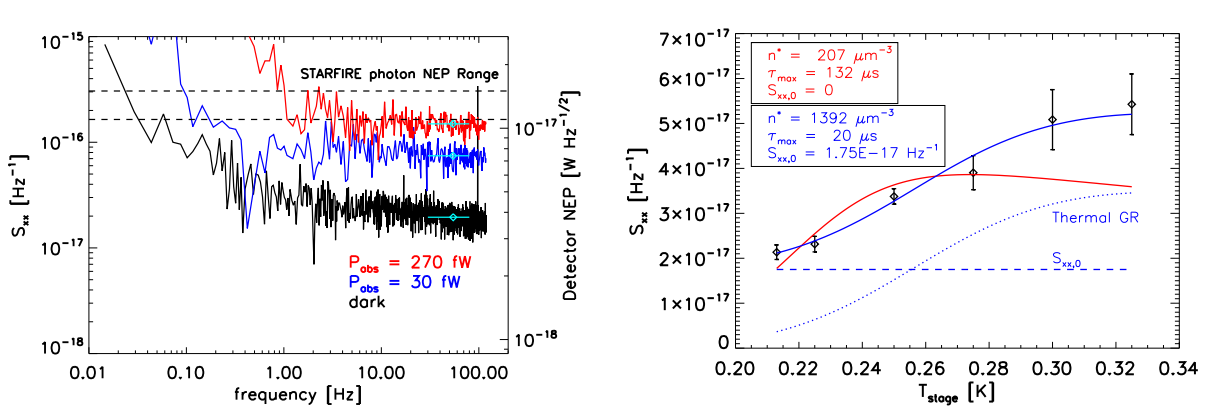

Fig. 4: Left Amplifier-subtracted $S_{\mathrm{xx}}$ for a representative KID measured dark (black), and with the cryogenic blackbody delivering $P_{\mathrm{abs}}=30 \mathrm{fW}$ (blue) and $270 \mathrm{fW}$ (red). White noise is estimated by averaging over $30-80 \mathrm{~Hz}$ (cyan), and conversion to a detector NEP uses the responsivity $R=1.2 \times 10^{9} \mathrm{~W}^{-1}$ measured at the lowest optical loading $P_{\mathrm{abs}}=20 \mathrm{fW}$. Right Amplifier-subtracted Sxx measured dark at various stage temperatures, averaged over 6 KIDs. Fits to Equation 4 are shown with $S_{x x, 0}=0(r e d)$ and $S_{x x, 0}$ a free parameter (blue solid), with the latter decomposed into thermal GR (blue dotted) and fixed noise floor (blue dashed). (Color figure online.)

where $n_{\gamma}$ is the photon occupation number in the detector, the thermal generation rate is $\Gamma_{\text {th }}=$ $\left(n_{\mathrm{th}} V / 2\right)\left(\tau_{\max }^{-1}+\tau_{\mathrm{th}}^{-1}\right), \tau_{\mathrm{th}}$ is the quasiparticle lifetime when $n_{\mathrm{qp}}=n_{\mathrm{th}}$, and the recombination rate is $\Gamma_{r}=\left(n_{\mathrm{qp}} V / 2\right)\left(\tau_{\max }^{-1}+\tau_{\mathrm{qp}}^{-1}\right)[11]$. In the limit of no optical loading this becomes:

$$
S_{x x} \rightarrow\left(\frac{\alpha \gamma S_{2}}{4 N_{0} \Delta_{0}}\right)^{2} \frac{4 n_{\mathrm{th}} \tau_{\mathrm{th}}}{V}\left(1+\frac{\tau_{\mathrm{th}}}{\tau_{\max }}\right)+S_{x x, 0}
$$

The temperature dependence of Equation 4 is dominated by the $n_{\mathrm{th}} \tau_{\mathrm{th}}\left(1+\tau_{\mathrm{th}} / \tau_{\mathrm{max}}\right)$ product. At low temperatures this term reduces to $2 n_{\text {th }} \tau_{\max }$ and increases rapidly with temperature, while at high temperatures this term asymptotes to $n^{*} \tau_{\max }$ [6]. In Figure 4 we show the white noise as a function of temperature, averaged over a subset (6/11) of the KIDs with the lowest amplifier noise. We show a fit to the data with fixed $P_{\mathrm{abs}}=S_{x x, 0}=0$, and $n^{*}$ and $\tau_{\max }$ as free parameters. This fit is poor, but the data are well reproduced by introducing a noise floor $S_{x x, 0} \approx 1.8 \times 10^{-17} \mathrm{~Hz}^{-1}$, or alternatively by assuming a frequency-dependent stray power $P_{\text {abs }} \approx 20(100 \mathrm{GHz} / v)$ fW for a minimum pair-breaking frequency of $100 \mathrm{GHz}$. These fits indicate thermal generation-recombination $(\mathrm{GR})$ noise is subdominant to other noise sources at our $210 \mathrm{mK}$ operating temperature, but dominates at $T>250 \mathrm{mK}$.

\subsection{Optical Measurements}

In a second test we expose the detector package to the cryogenic blackbody, and measure the frequency response and noise as a function of the blackbody temperature for $T_{\mathrm{BB}}=6.2-11$ $\mathrm{K}$. We compute the power incident on the feedhorn aperture by integrating the blackbody source function over the filter transmission profiles, assuming a single spatial mode and dual polarization response. Figure 5 shows the fractional frequency response and white noise for a representative channel. The slope of the response curve is the product of the responsivity and optical efficiency $\left(\eta_{\text {opt }}\right)$ :

$$
\frac{\delta x}{\delta P_{\mathrm{inc}}}=\frac{\delta x}{\delta P_{\mathrm{abs}}} \eta_{\mathrm{opt}}=\frac{\alpha \gamma S_{2}}{4 N_{0} \Delta_{0}} \frac{\eta_{\mathrm{pb}} \tau_{\mathrm{qp}}}{\Delta_{0} V} \eta_{\mathrm{opt}},
$$



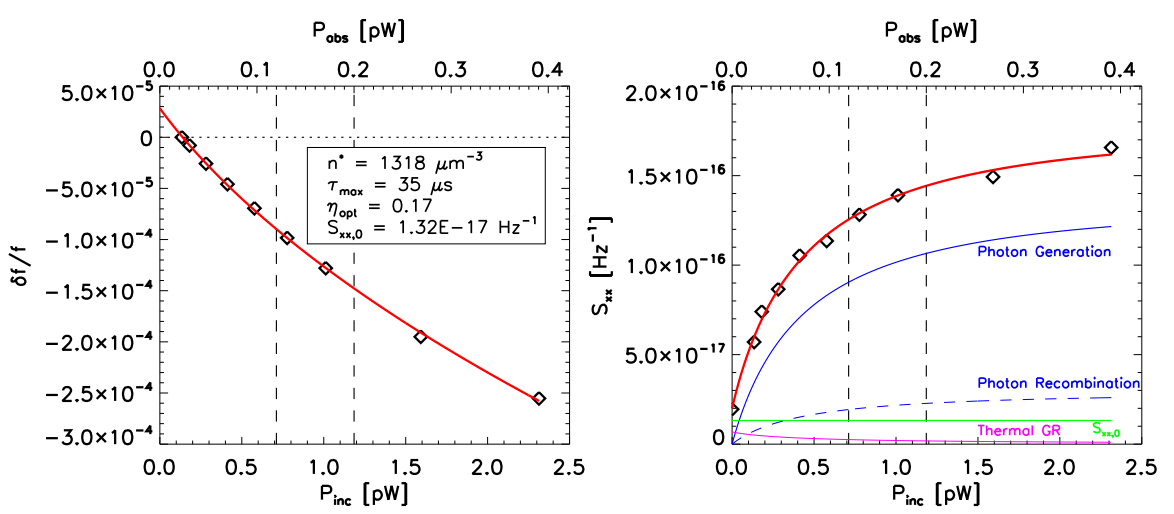

Fig. 5: Left Fractional frequency shift vs. $P_{\text {inc }}$ with model fit (red). Right $S_{\mathrm{xx}}$ vs. $P_{\text {inc }}$ with model fit (red), decomposed into contributions from photon generation noise (blue solid), photon recombination noise (blue dashed), thermal GR noise (magenta), and fixed noise floor (green). Vertical dashed lines in both figures mark the minimum and median STARFIRE optical loading of $120 \mathrm{fW}$ and $200 \mathrm{fW}$, respectively. (Color figure online.)

and the flattening of this curve at high $P_{\text {inc }}$ is consistent with the expected decrease in $\tau_{\mathrm{qp}}$ at large $n_{\mathrm{qp}}$.

We see an increase in white noise with $P_{\text {inc }}$ that we attribute to photon noise (Figure 4). The contribution of photon noise to $S_{\mathrm{xx}}$ is:

$$
S_{\mathrm{xx}, \gamma}=\left(\frac{\delta x}{\delta P_{\mathrm{inc}}}\right)^{2} \frac{2 h v P_{\mathrm{inc}}}{\eta_{\mathrm{opt}}}\left(1+\frac{2 \Delta_{0}}{h v \eta_{\mathrm{pb}}}\right),
$$

where we have neglected wave noise (negligible for $T_{\mathrm{BB}} \leq 11 \mathrm{~K}$ ), and the last term accounts for the recombination noise associated with optically-generated quasiparticles. With an assumption of $\eta_{\mathrm{pb}}=0.57$ this term is 1.21 , and Equation 6 then demonstrates how the ratio of $S_{\mathrm{xx}, \gamma}$ and $\left(\delta x / \delta P_{\text {inc }}\right)^{2} P_{\text {inc }}$ may be used to estimate $\eta_{\text {opt }}$.

We fit the response and noise data shown in Figure 5 using our full resonator model (Equations 1-3), with free parameters $n^{*}, \tau_{\mathrm{max}}, \eta_{\mathrm{opt}}$, and $S_{\mathrm{xx}, 0}$. The data are well fit with this model, and we find median values of $n^{*}=1240 \mu \mathrm{m}^{-3}, \tau_{\max }=35 \mu \mathrm{s}, \eta_{\mathrm{opt}}=0.17$, and $S_{x x, 0}=1.2 \times 10^{-17} \mathrm{~Hz}^{-1}$. This value of $\tau_{\max }$ is shorter than reported by other groups for aluminum [7, 4]. Our aluminum is currently deposited through a sputter deposition technique; a future shift to electron beam evaporation may produce higher purity films and longer quasiparticle lifetime, increasing the responsivity [1]. This optical efficiency is close to the $0.20-0.22$ range estimated from simulations of the coupling efficiency between the waveguide and absorber (Figure 1). The inferred responsivity at our lowest optical loading $\left(P_{\mathrm{abs}}=20 \mathrm{fW}\right)$ averaged over the array is $\delta x / \delta P_{\mathrm{abs}}=1.2 \times 10^{9} \mathrm{~W}^{-1}$. We combine this with our dark noise measurements to obtain detector NEPs, finding a median value of $4 \times 10^{-18}$ $\mathrm{W} \mathrm{Hz}^{-1 / 2}$ (Figure 4).

The STARFIRE arrays will operate with a typical optical loading of $200 \mathrm{fW}$, at a 250 $\mathrm{mK}$ base temperature. Increasing the optical load from $20 \mathrm{fW}$ to $200 \mathrm{fW}$ decreases the responsivity in our prototype KIDs by $\approx 40 \%$ (Figure 5 ). Additionally, the dark noise at 250 $\mathrm{mK}$ is $\approx 1.5$ times larger than at $210 \mathrm{mK}$ (Figure 4). Combined, this indicates an NEP of $8 \times$ $10^{-18} \mathrm{~W} \mathrm{~Hz}^{-1 / 2}$ with the optical load and operating temperature envisioned for STARFIRE, less than the photon NEP of $1.5 \times 10^{-17} \mathrm{~W} \mathrm{~Hz}^{-1 / 2}$. 


\section{Summary}

We have fabricated and characterized a 45-pixel STARFIRE prototype detector array. These LEKIDs are low volume $\left(76 \mu \mathrm{m}^{3}\right)$ devices fabricated with a single layer of $40 \mathrm{~nm}$ thick aluminum, are sensitive to both polarizations, and couple to free space with circular waveguide and conical feedhorns. Operating at $210 \mathrm{mK}$ we measure a typical NEP of $4 \times 10^{-18}$ $\mathrm{W} \mathrm{Hz}^{-1 / 2}$, and confirm that thermal GR noise is not the dominant noise source. With a 250 $\mathrm{mK}$ operating temperature and under a $200 \mathrm{fW}$ load, as we anticipate for STARFIRE, the detector NEP will be $\approx 8 \times 10^{-18} \mathrm{~W} \mathrm{~Hz}^{-1 / 2}$. This compares favorably to the typical photon NEP of $1.5 \times 10^{-17} \mathrm{~W} \mathrm{~Hz}^{-1 / 2}$.

Acknowledgements ACMB's work was supported by a NASA Space Technology Research Fellowship. Detector development for STARFIRE is supported by NASA grant 15-APRA15-0081. We thank C. Groppi for generously providing the tool used to drill the feedhorns.

\section{References}

1. R. Barends, S. van Vliet, J. J. A. Baselmans, S. J. C. Yates, J. R. Gao, and T. M. Klapwijk. Enhancement of quasiparticle recombination in Ta and Al superconductors by implantation of magnetic and nonmagnetic atoms. Phys. Rev. B, 79(2):020509, Jan. 2009. doi: 10.1103/PhysRevB.79.020509.

2. A. Barlis, S. Hailey-Dunsheath, C. M. Bradford, C. McKenney, H. G. Le Duc, and J. Aguirre. Development of low-noise kinetic inductance detectors for far-infrared astrophysics. In APS April Meeting Abstracts, Jan. 2017.

3. P. Barry. J. Low Temp. Phys. In This Special Issue, 2017.

4. J. J. A. Baselmans, J. Bueno, S. J. C. Yates, O. Yurduseven, N. Llombart, K. Karatsu, A. M. Baryshev, L. Ferrari, A. Endo, D. J. Thoen, P. J. de Visser, R. M. J. Janssen, V. Murugesan, E. F. C. Driessen, G. Coiffard, J. Martin-Pintado, P. Hargrave, and M. Griffin. A kilo-pixel imaging system for future space based far-infrared observatories using microwave kinetic inductance detectors. $A \& A, 601: A 89$, May 2017. doi: 10.1051/0004-6361/201629653.

5. J. Gao, J. Zmuidzinas, A. Vayonakis, P. Day, B. Mazin, and H. Leduc. Equivalence of the Effects on the Complex Conductivity of Superconductor due to Temperature Change and External Pair Breaking. Journal of Low Temperature Physics, 151:557-563, Apr. 2008. doi: 10.1007/s10909-007-9688-z.

6. P. D. Mauskopf, S. Doyle, P. Barry, S. Rowe, A. Bidead, P. A. R. Ade, C. Tucker, E. Castillo, A. Monfardini, J. Goupy, and M. Calvo. Photon-Noise Limited Performance in Aluminum LEKIDs. Journal of Low Temperature Physics, 176:545-552, Aug. 2014. doi: 10.1007/s10909-013-1069-1.

7. H. McCarrick, D. Flanigan, G. Jones, B. R. Johnson, P. Ade, D. Araujo, K. Bradford, R. Cantor, G. Che, P. Day, S. Doyle, H. Leduc, M. Limon, V. Luu, P. Mauskopf, A. Miller, T. Mroczkowski, C. Tucker, and J. Zmuidzinas. Horn-coupled, commercially-fabricated aluminum lumped-element kinetic inductance detectors for millimeter wavelengths. Review of Scientific Instruments, 85(12):123117, Dec. 2014. doi: $10.1063 / 1.4903855$.

8. C. M. McKenney, H. G. Leduc, L. J. Swenson, P. K. Day, B. H. Eom, and J. Zmuidzinas. Design considerations for a background limited 350 micron pixel array using lumped element superconducting microresonators. In Society of Photo-Optical Instrumentation 
Engineers (SPIE) Conference Series, volume 8452 of Society of Photo-Optical Instrumentation Engineers (SPIE) Conference Series, Sept. 2012. doi: 10.1117/12.925759.

9. M. Meixner, A. Cooray, R. Carter, M. DiPirro, A. Flores, D. Leisawitz, L. Armus, C. Battersby, E. Bergin, C. M. Bradford, K. Ennico, G. J. Melnick, S. Milam, D. Narayanan, K. Pontoppidan, A. Pope, T. Roellig, K. Sandstrom, K. Y. L. Su, J. Vieira, E. Wright, J. Zmuidzinas, S. Alato, S. Carey, M. Gerin, F. Helmich, K. Menten, D. Scott, I. Sakon, and R. Vavrek. The Far-Infrared Surveyor Mission study: paper I, the genesis. In Space Telescopes and Instrumentation 2016: Optical, Infrared, and Millimeter Wave, volume 9904 of Society of Photo-Optical Instrumentation Engineers (SPIE) Conference Series, page 99040K, July 2016. doi: 10.1117/12.2240456.

10. B. D. Uzgil, J. E. Aguirre, C. M. Bradford, and A. Lidz. Measuring Galaxy Clustering and the Evolution of [C II] Mean Intensity with Far-IR Line Intensity Mapping during $0.5<\mathrm{z}<1.5$. ApJ, 793:116, Oct. 2014. doi: 10.1088/0004-637X/793/2/116.

11. J. Zmuidzinas. Superconducting microresonators: Physics and applications. Annual Review of Condensed Matter Physics, 3(1):169-214, 2012. doi: 10. 1146/annurev-conmatphys-020911-125022. URL http://dx.doi.org/10.1146/ annurev-conmatphys-020911-125022 